To appear in Computer Methods in Biomechanics and Biomedical Engineering: Imaging 6 Visualization Vol. 00, No. 00, Month 20XX, 2-12

\title{
Performance analysis of speckle ultrasound image filtering
}

\author{
R. Rosa and F. C. Monteiro* \\ Polytechnic Institute of Bragança, \\ Campus Santa Apolónia, Bragança, Portugal
}

(Received 00 Month 20XX; accepted 00 Month 20XX)

\begin{abstract}
Over the last three decades, several despeckling filters have been developed by researchers to reduce the speckle noise inherently present in ultrasound B-scan images without losing the diagnostic information. This paper compiles and compares well-known techniques mostly used in the smoothing or suppression of speckle noise in ultrasound images. A comparison of the methods studied is done based on an experiment, using quality metrics, texture analysis and interpretation of row profiles to evaluate their performance and show the benefits each one can contribute to denoising and feature preservation. To test the methods, a noise-free image of a kidney is used and then the Field II program simulates a B-mode ultrasound image. This way, the smoothing techniques can be compared using numeric metrics, taking the noise-free image as a reference. In this study, a total of seventeen different speckle reduction algorithms have been documented based on spatial filtering, diffusion filtering and wavelet filtering, with fifteen qualitative metrics estimation. We use the tendencies observed in our study in real images. A new evaluation metric is proposed to evaluate the despeckling results.
\end{abstract}

Keywords: Speckle noise, speckle reduction, ultrasound images, quality metrics.

\section{Introduction}

Image quality metrics are figures of merit used for ultrasound speckle reduction evaluation. In this study we consider several image quality metrics and study their behavior when evaluating various ultrasound despeckling filters.

Medical ultrasound imaging is a technique that has become much more widespread than other medical imaging techniques since this technique is more accessible, less expensive, safe, simpler to use and produces images in real-time. However, ultrasound images are degraded by an intrinsic artifact called 'speckle', which is the result of the constructive and destructive coherent summation of ultrasound echoes (Ortiz 2012).

Speckle noise in ultrasound biomedical B-scan images is a random granular pattern produced mainly by multiplicative noise that degrades the visual evaluation in ultrasound imaging and does not correspond to the actual tissue microstructure and tends to mask the presence of low-contrast lesions and fine structures (Wagner 1983) as shown in Figure 1. It is generated by the fact that there are a number of elementary scatterers within each resolution cell of the image that reflect the incident wave back towards the ultrasound sensor. The backscattered coherent waves with different phases undergo constructive and destructive interferences in a random manner.

Removing noise from the original image is still a challenging research in image processing (Ortiz 2012; Huang 2013). The presence of speckle noise severely degrades the signal-to-noise ratio (SNR) and contrast resolution of the image, making human interpretation and computer assisted detection techniques difficult and inconsistent. Therefore, a speckle reduction process is quite necessary in low

*Corresponding author. Email: monteiro@ipb.pt 


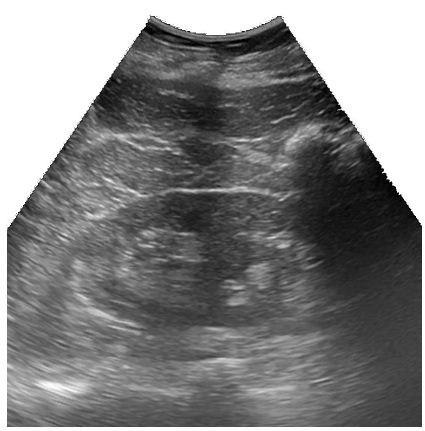

(a)

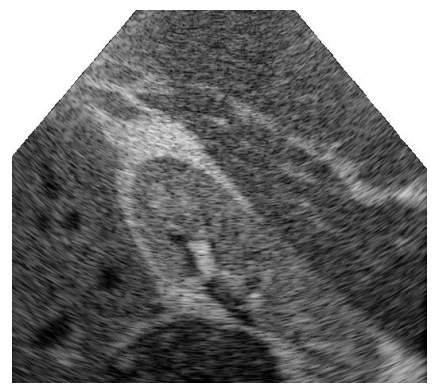

(b)

Figure 1. Speckle noise. (a) Real ultrasound image. (b) Simulated ultrasound image.

SNR, low contrast ultrasound images for enhancing visualization of organ anatomy and improving the accuracy of object detection without affecting the important diagnostic features of the image (Finn 2011).

A smoothing of speckle and preservation of edges are in a general sense divergent. A trade-off between noise reduction and the preservation of the actual image features and contrast has to be made in order to enhance the relevant image content for diagnostic purposes. Best contrast is meant in the sense of decreasing the variance in a homogeneous region while distinct regions are well defined.

Thakur and Anand (Thakur 2005) presented a comparative study of various wavelet filter based denoising methods according to different thresholding values applied to ultrasound images. In (Mateo 2009), the authors investigate some of the techniques mostly used in the smoothing or suppression of speckle noise in ultrasound images. In a recent paper (Finn 2011) a detailed description and comparison of speckle reduction of medical ultrasound, and in particular echocardiography, is presented using fifteen speckle reduction filters and five quality metrics. Although they used simulated images, the speckle noise used on those images is not consistent with the predominate noise that is presented in ultrasound images. To a more realistic simulation of ultrasound speckle noise, the Field II program (Jensen 2004) is used to simulate B-mode ultrasound images. This model takes into account the effects of frequency-dependent attenuation, backscattering, and dispersion.

This article investigates and compiles well-known techniques used in the smoothing or suppression of speckle noise in ultrasound images. A comparison of the methods studied is done based in real ultrasound images and in computer simulated images, using quality metrics to test their performance and show the benefits each one can contribute. A new evaluation metric is proposed based on local similarity map and edge preservation.

\section{Speckle Filtering Techniques}

As a primary low-level image processing procedure, speckle noise removal has been extensively studied and many denoising algorithms have been proposed. They are divided mainly into three classes: (i) techniques that are applied directly in the original image, (ii) techniques based on anisotropic diffusion and (iii) techniques that are applied in the wavelet domain.

\subsection{Adaptive local filters}

Adaptive filters take a moving filter window and estimate the statistical characteristics of the image inside the filter region, such as the local mean and the local variance. Spatial adaptive filters like median, (Lee 1980), (Frost 1982) and (Kuan 1985) filters assume that the speckle noise is essentially a multiplicative noise. Wiener filter (Jin 2003) performs smoothing of the image based 
on the computation of local image variance. Ideal Fourier and Butterworth filtering performs image enhancement by applying the filter function and inverse FFT on the image (Loizou 2008). Bilateral filtering technique (Tomasi 1998), basically is a combination of a spatial and range filter, where each output pixel value is a Gaussian weighted average of its neighbours in both space and intensity range. This nonlinear combination of nearby pixel values, gives the well-known good performance of this filter in smoothing while preserving edges.

\subsection{Anisotropic diffusion filters}

Diffusion filters remove noise from an image by modifying the image via solving a partial differential equation ( $\mathrm{Yu}$ 2010; Deng 2011). Speckle reducing filters based on anisotropic diffusion algorithms were introduced by Perona and Malik (Perona 1990) (PMAD). Unlike conventional spatial filtering techniques that do not protect region boundaries or small structures, anisotropic diffusion techniques can simultaneously eliminate noise and preserve or even enhance edges (Perona 1990). Because of this attractive feature, many researchers have applied anisotropic diffusion techniques in speckle noise reduction. Weickert (Weickert 1999) introduced the coherence enhancing diffusion (CED), that allows the level of smoothing to vary directionally by a tensor-valued diffusion function. Yu and Acton ( $\mathrm{Yu}$ 2002) first introduced partial differential equation by integrating the spatially adaptive (Lee 1980) filter and the Perona-Malik diffusion, which they called Speckle Reducing Anisotropic Diffusion (SRAD). SRAD provides significant improvement in speckle suppression and edge preservation when compared to traditional methods like Lee, Frost and Kuan filters. In ( $\mathrm{Fu} 2005)$ is proposed the edge enhanced anisotropic diffusion (EEAD) method that includes anisotropic diffusion and edge enhancement. Krissian et al. (Krissian 2007) proposed the oriented speckle reducing anisotropic diffusion (OSRAD) filter which allows different levels of filtering across the image contours and in the principal curvature directions.

\subsection{Wavelet filters}

Wavelet transform, unlike Fourier transform, shows localization in both time and frequency and it has proved itself to be an efficient tool for noise removal (Khare 2010). One widespread method exploited for speckle reduction is wavelet shrinkage, including VisuShrink (Donoho 1994), SureShrink (Donoho 1995) and BayeShrink (Chang 2000). A wavelet-based multiscale linear minimum mean square-error estimation (LMMSE) is proposed in (Zhang 2005), where an interscale model, the wavelet coefficients with the same spatial location across adjacent scales, was combined as a vector, to which the LMMSE in then applied.

\section{Image Quality Evaluation Metrics}

As shown above, a large variety of speckle reduction filters have been proposed. Evaluating the relative performance of these filters has been obtained by a number of different methods.

Common techniques for evaluating relative performance are quantitative image quality metrics, and qualitative inspection, often by the authors themselves. A great deal of effort has been made in recent years to develop objective image quality metrics that correlate with perceived quality measurement. Test data for evaluation includes clinical and phantom images, as well as simulated ultrasound which allows evaluation of filtering relative to an ideal speckle free reference. However, the measurement of ultrasound image enhancement is difficult and there is no unique algorithm available to measure enhancement of ultrasound image (Wang 2011).

In this study, resulting images were evaluated using several quality evaluation metrics such as average difference (AD), Pratt's figure of merit (FOM), root mean square error (RMSE), signal to noise ratio (SNR), peak signal to noise ratio (PSNR), maximum difference (MD), normalized 
absolute error (NAE), normalized cross-correlation (NK), structural content (SC), coefficient of correlation ( $\mathrm{CoC})$, universal quality index (UQI), quality index based on local variance (QILV), Laplacian mean square error (LMSE), mean structural similarity quality index (MSSIM). This last metric is not just an index, as it includes a visibility error map for viewing areas where both original image and distorted image are different. We also propose a new metric which combines the edge preservation evaluation with a local similarity map to obtain the Speckle Reduction Score (SRS).

Consider $f(i, j)$ as the original image and $g(i, j)$ as the filtered image, with $m \times n$ size.

- Signal to noise ratio (SNR): A common way to evaluate the noise suppression in the case of multiplicative noise in coherent imaging. It compares the level of desired signal with respect to the level of background noise

$$
S N R=10 \log _{10} \frac{\sum f(i, j)^{2}}{\sum(f(i, j)-g(i, j))^{2}}
$$

- Root mean square error (RMSE): It is the root average of the square of the difference between original and the denoised image intensity values divided by the size of the image

$$
R M S E=\sqrt{\frac{\sum(f(i, j)-g(i, j))^{2}}{m \times n}}
$$

A higher/lower RMSE value corresponds to maximum/minimum difference between the original and the denoised image;

- Peak signal-to-noise ratio (PSNR): It provides the quality of the image in terms of a ratio between the maximum possible power of the signal and the denoised image

$$
P S N R=20 \log _{10} \frac{255}{R M S E}
$$

Higher PSNR value shows better image quality;

- Pratt's figure of merit (FOM) (Pratt 2007): It measures the performance of edge preservation of the image

$$
F O M=\frac{1}{\max \left(E_{I}, E_{F}\right)} \sum_{i=1}^{E_{F}} \frac{1}{1+\alpha d_{i}^{2}}
$$

where $E_{I}$ and $E_{F}$ are the number of ideal and actual filtered edge pixels. $d_{i}$ is the Euclidean distance between the $i$-th detected edge pixel and the nearest ideal edge pixel, and $\alpha$ is a constant typically set to $1 / 9$.

FOM strongly depends on the method used to obtain a binary edge map. Instead of using a different edge detector that maximizes FOM for each speckle reduction algorithm, we apply the same detector, the Canny detector, to provide a fair comparison of algorithms. FOM ranges between 0 and 1, with unity for the ideal performance on preserving edges;

- Normalized cross-correlation (NK): It is a correlation based image quality measure and its value is unity for identical images

$$
N K=\frac{\sum(f(i, j) \cdot g(i, j))}{\sum(f(i, j))^{2}}
$$

- Average difference $(\mathrm{AD})$ : It measures the average difference value between a particular pixel 
in the original and denoised image

$$
A D=\frac{\left|\sum(g(i, j)-f(i, j))\right|}{m \times n}
$$

- Maximum difference (MD): Maximum difference between the original and denoised image

$$
M D=\max (\max (g(i, j)-f(i, j)))
$$

- Normalized absolute error (NAE): It measures the error prediction accuracy of the image

$$
N A E=\frac{\sum a b s(g(i, j)-f(i, j))}{\sum a b s(f(i, j))}
$$

- Structural content (SC): Structural content is given by

$$
S C=\frac{\sum f(i, j)^{2}}{\sum g(i, j)^{2}}
$$

- Laplacian mean square error (LMSE): An important attribute of image quality is local contrast. The basic approach of evaluating image local contrast is the Laplacian mean square error

$$
L M S E=\frac{\sum\left(O P-4 \nabla^{2}(g(i, j))\right)^{2}}{\sum O P^{2}}
$$

where $O P$ is given by

$$
O P=4 \nabla^{2}(f(i, j))
$$

where $\nabla^{2}$ is the Laplace operator;

- Coefficient of correlation $(\mathrm{CoC})$ : Indicates the strength and direction of linear relationship between the original and denoised images

$$
C o C=\frac{\sum\left(f(i, j)-\mu_{f}\right) \cdot\left(g(i, j)-\mu_{g}\right)}{\sqrt{\sum\left(f(i, j)-\mu_{f}\right)^{2} \cdot \sum\left(g(i, j)-\mu_{g}\right)^{2}}}
$$

where $\mu_{f}$ and $\mu_{g}$ are the mean of the noise free and denoised image respectively.

- Universal quality index (UQI) (Wang 2002): It is designed by modeling any image distortion as a combination of three factors - loss of correlation, luminance distortion, and contrast distortion

$$
U Q I=\frac{4 \sigma_{f, g} \mu_{f} \mu_{g}}{\left(\sigma_{f}^{2}+\sigma_{g}^{2}\right) \cdot\left(\mu_{f}^{2}+\mu_{g}^{2}\right)}
$$

where $\mu$ and $\sigma^{2}$ are the mean intensity and the variance of each image and $\sigma_{f, g}$ is the covariance between them. UQI ranges between -1 and 1 , with unity for the best filtered image quality.

- Mean structural similarity index map (MSSIM) (Wang 2004): MSSIM is used to compare luminance, contrast and structure of two different images. It can be treated as a similarity 
measure between images. The MSSIM of two images can be calculated as

$$
M S S I M=\frac{\sum S S I M(f, g)}{m \times n}
$$

with SSIM as

$$
\operatorname{SSIM}(f, g)=\frac{\left(2 \mu_{f} \mu_{g}+C_{1}\right) \cdot\left(2 \sigma_{f, g}+C_{2}\right)}{\left(\mu_{f}^{2}+\mu_{g}^{2}+C_{1}\right) \cdot\left(\sigma_{f}^{2}+\sigma_{g}^{2}+C_{2}\right)}
$$

where $\mu$ and $\sigma^{2}$ are the mean intensity and the variance of each image, $\sigma_{f, g}$ is the covariance between them, and $C_{1}$ and $C_{2}$ are two constants to avoid instability when $\mu_{f}^{2}+\mu_{g}^{2}$ is very close to zero and are defined as $C_{i}=\left(255 \cdot k_{i}\right)^{2}$ in which $k_{i}<<1$. The MSSIM value should be closer to unity for optimal measure of similarity;

- Quality index based on local variance (QILV) (Aja-Fernandez 2006): It is based on the assumption that a great amount of the structural information of an image is coded in its local variance distribution.

$$
Q I L V(f, g)=\frac{2 \mu_{f} \mu_{g}}{\mu_{f}^{2}+\mu_{g}^{2}} \cdot \frac{2 \sigma_{f} \sigma_{g}}{\sigma_{f}^{2}+\sigma_{g}^{2}} \cdot \frac{\sigma_{f, g}}{\sigma_{f} \sigma_{g}}
$$

The first term in eq. (16) carries out a comparison between the mean of the local variance distributions of both images. The second one compares the standard deviation of the local variances. The third term is the one to introduce spatial coherence. To avoid some computational problems with small values, some constants may be added to every term.

Note that though there is a great similarity between eq. (16) and the SSIM index in eq. (15), the latter is the mean of the local statistics of the images, and the former deals with the global statistics of the local variances of the images.

- Speckle reduction score (SRS): Although the MSSIM index has shown to be a very useful index in many experiments, cases may arise in which the quality measure obtained does not match properly a subjective judgment based on the visual information. We propose a new metric, Speckle Reduction Score (SRS), which computation consists of two stages. In the first stage, the local similarity map is computed, and then in the second stage, we pool the similarity map into a single similarity score.

$$
S R S=\frac{\left(2 \mu_{f} \mu_{g}+C_{1}\right) \cdot\left(2 \sigma_{f, g}+C_{2}\right)}{\left(\mu_{f}^{2}+\mu_{g}^{2}+C_{1}\right) \cdot\left(\sigma_{f}^{2}+\sigma_{g}^{2}+C_{2}\right)} \cdot \frac{\sum\left(\nabla^{2} f-\nabla^{2} g\right)^{2}}{\sum\left(\nabla^{2} f\right)^{2}}
$$

where $C_{1}$ and $C_{2}$ are positive constants to increase the stability of SRS and $\nabla^{2}$ is the Laplace operator.

\section{Implementation of Despeckling Algorithms and Results}

In this section, the performance of applying the seventeen despeckling filters, described in Section 2 , on simulated data and on real ultrasound images, is evaluated with fifteen quantitative metrics.

\subsection{Experiments with synthetic images}

To evaluate the effects of denoising filters on noisy images it is necessary to have reference images (without noise or with low noise level) used to compare the output of filtering and quantify the 


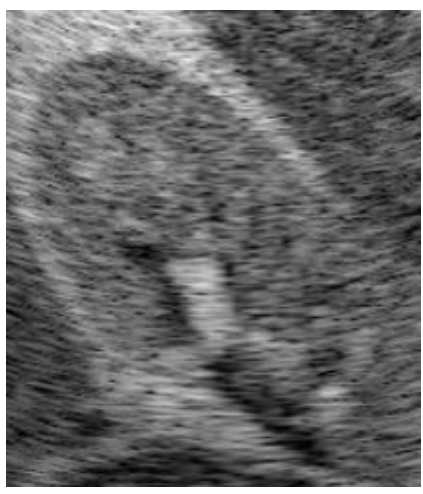

(a)

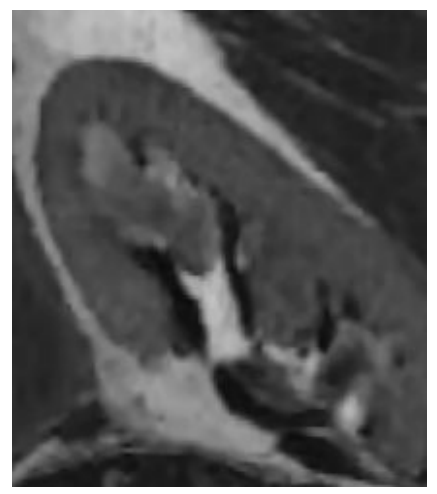

(b)

Figure 2. Simulated speckle noise. (a) Noisy Field II image. (b) Reference MRI image for the comparison.

Table 1. Image quality evaluation metrics computed for the 17 despeckling filters. The arrow under each metric indicates the expected tendency for the best despeckling filters.

\begin{tabular}{|c|c|c|c|c|c|c|c|c|c|c|c|c|c|c|c|}
\hline Metrics & $\begin{array}{c}\text { RMSE } \\
\downarrow \\
\end{array}$ & $\begin{array}{c}\text { SNR } \\
\uparrow \\
\end{array}$ & $\begin{array}{c}\text { PSNR } \\
\uparrow \\
\end{array}$ & $\begin{array}{c}\text { LMSE } \\
\uparrow \\
\end{array}$ & $\begin{array}{c}\text { MD } \\
\downarrow \\
\end{array}$ & $\begin{array}{c}\mathrm{AD} \\
\downarrow \\
\end{array}$ & $\begin{array}{c}\text { NK } \\
\uparrow \\
\end{array}$ & $\begin{array}{c}\mathrm{CoC} \\
\uparrow \\
\end{array}$ & $\begin{array}{c}\text { NAE } \\
\downarrow\end{array}$ & $\begin{array}{c}\text { UQI } \\
\uparrow \\
\end{array}$ & $\begin{array}{c}\text { QILV } \\
\uparrow \\
\end{array}$ & $\begin{array}{c}\text { FOM } \\
\uparrow \\
\end{array}$ & $\begin{array}{c}\mathrm{SC} \\
\downarrow \\
\end{array}$ & $\begin{array}{c}\text { MSSIM } \\
\uparrow \\
\end{array}$ & $\begin{array}{c}\text { SRS } \\
\uparrow \\
\end{array}$ \\
\hline Noise & 38.82 & 10.84 & 16.35 & 1.14 & 154 & 22.23 & 0.77 & 0.67 & 0.32 & 0.09 & 0.73 & 0.21 & 1.49 & 0.20 & 0.22 \\
\hline Median & 34.46 & 11.80 & 17.38 & 3.61 & 139 & 22.37 & 0.79 & 0.76 & 0.29 & 0.15 & 0.74 & 0.44 & 1.45 & 0.47 & 1.68 \\
\hline Lee & 33.28 & 12.03 & 17.74 & 27.43 & 121 & 22.31 & 0.79 & 0.76 & 0.32 & 0.17 & 0.73 & 0.36 & 1.47 & 0.53 & 14.54 \\
\hline Frost & 33.37 & 12.03 & 17.66 & 43.32 & 120 & 22.08 & 0.80 & 0.79 & 0.29 & 0.16 & 0.75 & 0.48 & 1.42 & 0.53 & 23.09 \\
\hline Kuan & 34.06 & 11.86 & 17.49 & 2.65 & 121 & 21.82 & 0.79 & 0.76 & 0.29 & 0.14 & 0.75 & 0.38 & 1.43 & 0.44 & 1.17 \\
\hline Wiener & 34.12 & 11.85 & 17.47 & 2.53 & 128 & 21.84 & 0.79 & 0.76 & 0.29 & 0.15 & 0.75 & 0.33 & 1.43 & 0.47 & 1.18 \\
\hline Fourier & 34.96 & 11.65 & 17.26 & 15.57 & 138 & 21.51 & 0.79 & 0.74 & 0.30 & 0.14 & 0.73 & 0.45 & 1.44 & 0.42 & 6.47 \\
\hline Butterworth & 34.29 & 11.80 & 17.43 & 14.76 & 131 & 21.43 & 0.79 & 0.75 & 0.29 & 0.15 & 0.74 & 0.45 & 1.43 & 0.45 & 6.69 \\
\hline Bilateral & 33.42 & 12.02 & 17.65 & 47.20 & 122 & 22.25 & 0.80 & 0.79 & 0.29 & 0.17 & 0.75 & 0.55 & 1.43 & 0.54 & 25.38 \\
\hline PMAD & 33.19 & 12.06 & 17.71 & 44.29 & 122 & 21.70 & 0.80 & 0.78 & 0.29 & 0.16 & 0.75 & 0.36 & 1.42 & 0.54 & 23.69 \\
\hline CED & 35.72 & 11.50 & 17.07 & 2.33 & 130 & 22.22 & 0.78 & 0.73 & 0.30 & 0.12 & 0.74 & 0.24 & 1.46 & 0.33 & 0.76 \\
\hline SRAD & 38.35 & 11.18 & 16.46 & 41.31 & 134 & 28.88 & 0.75 & 0.78 & 0.31 & 0.16 & 0.72 & 0.33 & 1.63 & 0.50 & 20.62 \\
\hline EEAD & 34.09 & 11.84 & 17.48 & 2.80 & 131 & 21.32 & 0.80 & 0.75 & 0.29 & 0.15 & 0.75 & 0.44 & 1.42 & 0.45 & 1.25 \\
\hline OSRAD & 49.25 & 9.66 & 15.09 & 11.38 & 141 & 27.72 & 0.73 & 0.70 & 0.33 & 0.15 & 0.69 & 0.27 & 1.66 & 0.48 & 5.46 \\
\hline VisuShrink & 35.63 & 11.52 & 17.10 & 1.48 & 133 & 22.23 & 0.78 & 0.73 & 0.30 & 0.12 & 0.74 & 0.29 & 1.46 & 0.33 & 0.48 \\
\hline SureShrink & 33.65 & 11.99 & 17.59 & 48.63 & 139 & 22.20 & 0.79 & 0.78 & 0.29 & 0.18 & 0.75 & 0.47 & 1.44 & 0.52 & 25.39 \\
\hline BayeShrink & 33.65 & 11.99 & 18.71 & 47.06 & 137 & 22.21 & 0.79 & 0.78 & 0.29 & 0.18 & 0.75 & 0.46 & 1.44 & 0.52 & 24.52 \\
\hline LMMSE & 33.42 & 12.04 & 17.65 & 60.98 & 123 & 22.23 & 0.80 & 0.79 & 0.29 & 0.17 & 0.76 & 0.54 & 1.43 & 0.53 & 32.52 \\
\hline
\end{tabular}

improvement in image quality. Usually both noisy and reference images might be obtained with the same scanner and under the same running conditions. This is very difficult because of the highly operator dependence of the ultrasound exams and the random variation of scattering and speckle phenomena in each acquisition. In this case, conventional metrics cannot be used to indicate the quality obtained with speckle filtering. For this, it is useful to use synthetic images obtained for example by means of anatomic phantoms or by computer simulations for comparison. In our study, Field II (Jensen 2004) is used to simulate a B-mode ultrasound image (Figure 2.a) of an MRI noise-free image of a kidney as the reference image for filtering evaluation (Figure 2.b).

Table 1 summarizes the performance of the speckle filters, applied to the simulated image, through the calculation of several performance metrics. The best value for each metric is showed in bold. Some of the filtered images are shown in Figure 3. The profile for each image is obtained from column in white on the noise free image.

Although the metrics used in this study produce different scores for different levels of despeckling, the variability of the results is very low in most of them. The exceptions are LMSE, FOM, MSSIM and SRS. However, as FOM quantifies only the average distortion in edge pixel locations between each filtered image it does not evaluate the speckle reduction inside the regions.

The quantitative comparisons show that several filters can reduce speckle noise effectively in 

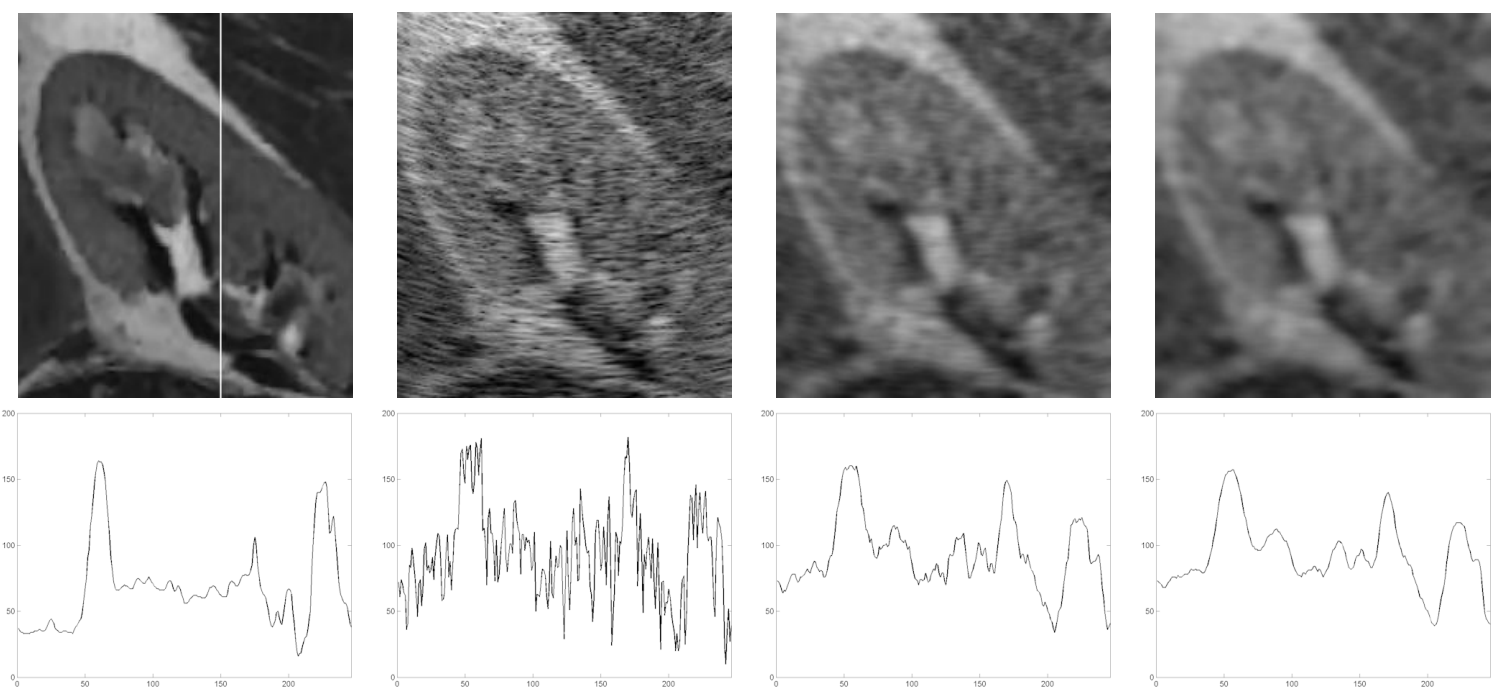

Noise free

Noise
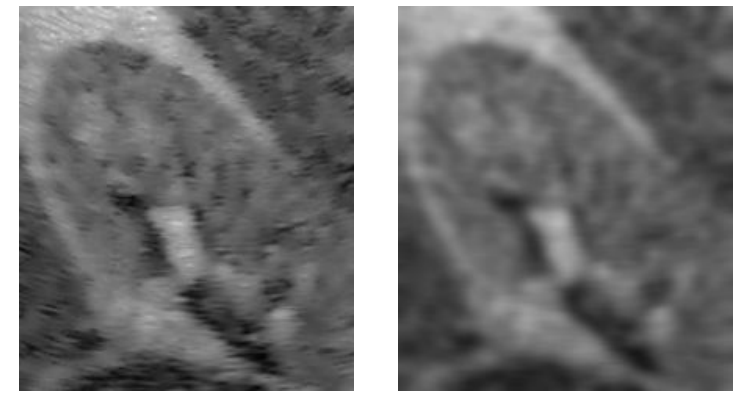

Lee

Frost
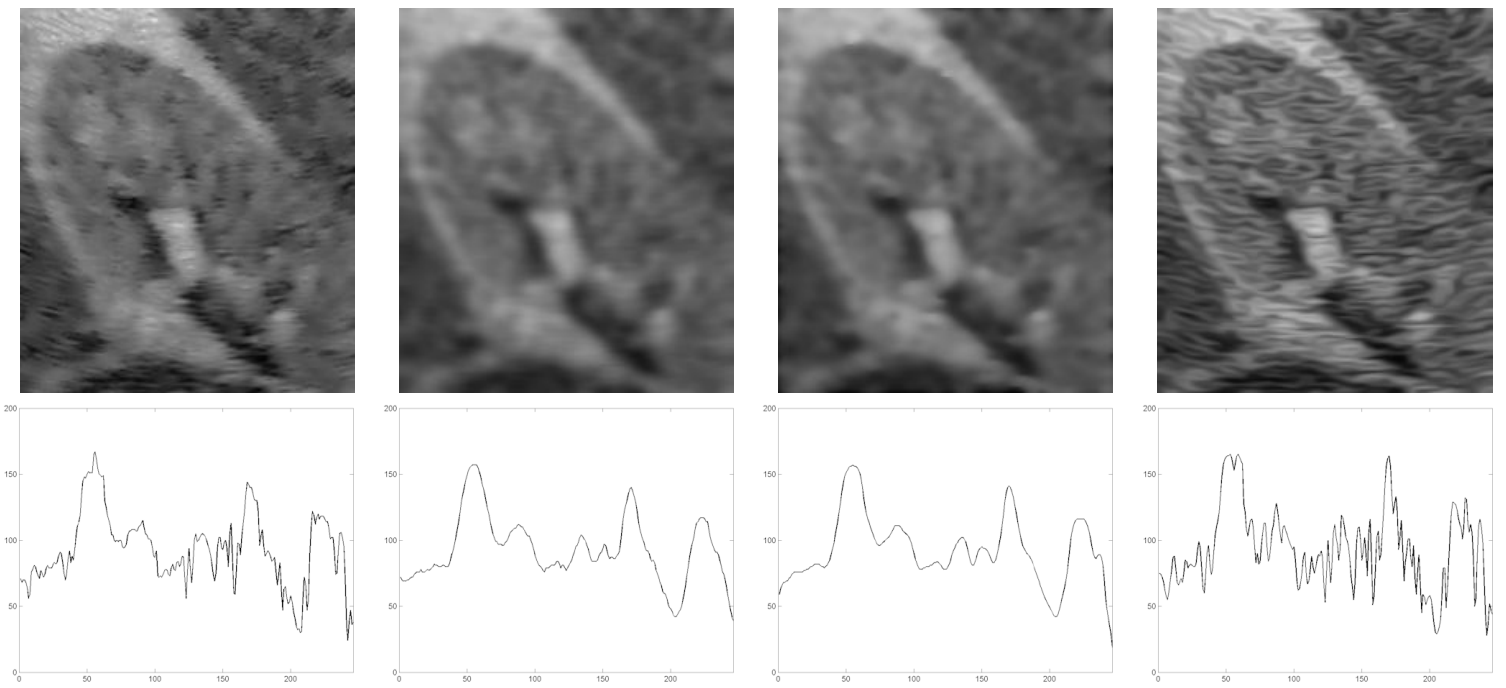

Kuan

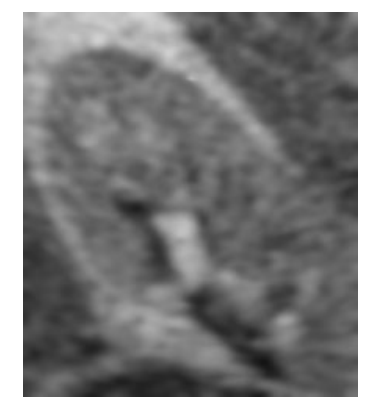

Bilateral

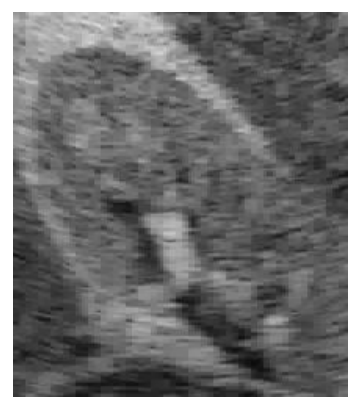

PMAD

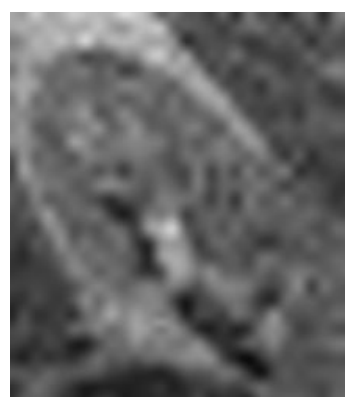

CED

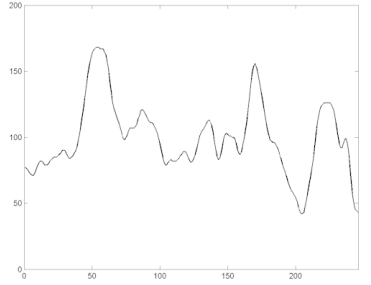

SRAD
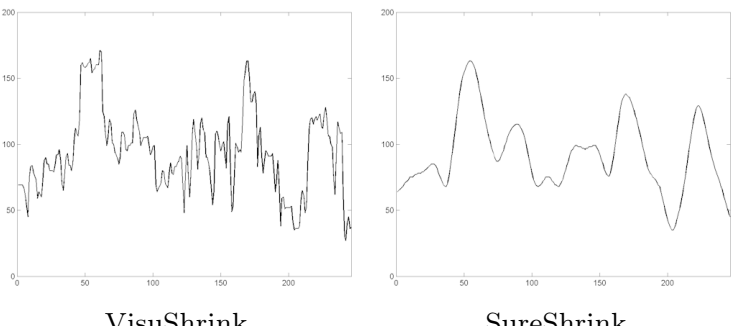

SureShrink

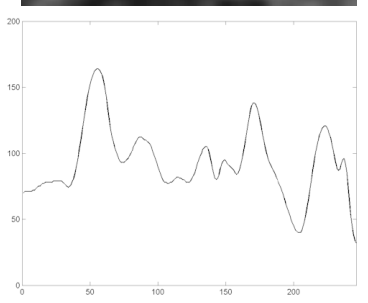

LMMSE

Figure 3. Filtering result comparison. The first two images are the noise free image and the simulated ultrasound speckle noise image. The other ones are filtered images with profiles generated by the despeckling filters described in Section 2 . 
terms of noise reduction, edge preservation, contrast preservation, and structure preservation. The output of the local adaptive filters vary in the speckle suppression level: the Lee, Frost and Bilateral remove most of the speckle pattern with some blurring. The Kuan, Wiener and Median filter remove considerably less of the speckle pattern. The anisotropic diffusion filters also exhibit a range of output quality. The PMAD and SRAD filters both display good speckle suppression. The CED filter displays artifacts, introduced by the enhancement of image contours (including those of the speckle texture). Finally, the wavelet filters output displays a good level of speckle reduction, the exception is the VisuShrink filter where some of the speckle pattern does still remain.

From Figure 3, we find that the speckle noise is reduced in homogeneous regions by almost every methods. The intensity variation caused by speckle in the background is still obvious in the images filtered by Kuan, CED and VisuShrink filters. The values obtained for the performance metric, indicate that the best despeckling filters are LMMSE, SureShrink, Bilateral, BayeShrink, PMAD and Frost. Filters Median, CED and VisuShrink exhibit poor performance results.

\subsection{Experiments on real ultrasound images}

Performance of a despeckling algorithm can also be subjectively measured by visual inspection of enhanced images by experts. For this purpose, we applied the different filters evaluated in the previous sections to real ultrasound images. The despeckling results and the profile of the white column, in the first image, are shown in Figure 4.

By visually inspecting the images and the column profiles it can be seen that the results with real ultrasound images are consistent with the evaluation made with simulated images. The Kuan, CED and VisuShrink filters output still contains some speckle noise. In general, the filters have produced good results in maintaining the edges, although in Sureshrink we can see some smoothing.

\section{Conclusion}

This paper compares some of the different algorithms and methods currently used to smooth speckle noise in medical ultrasound images. A new evaluation metric is proposed to evaluate the despeckling results. A comparative study of noise suppression methods in ultrasound images was carried out on a noise-free synthetic image of a kidney. We have used the Field II software to corrupt the image, adding the typical noise in ultrasound images. Afterwards we have shown some of the most common smoothing techniques over this image using numeric metrics, taking the noise-free image as a reference. In this study, a total of seventeen different speckle reduction algorithms have been documented based on spatial filtering, diffusion filtering and wavelet filtering, with fifteen qualitative metrics estimation. At the second stage the effects of applying the speckle filtering techniques were tested on data acquired in real ultrasound images.

\section{References}

Aja-Fernandez S, San Jose Estepar R, Alberola Lopez C, Westin CF. 2006. Image quality assessment based on local variance. In 28th IEEE EMBS Annual International Conference, p. 4815-4818.

Chang S, Yu B, Vetterli M. 2000. Adaptive wavelet thresholding for image denoising and compression. IEEE Transactions on Image Processing 9(9):1532-1546.

Deng Y, Wang Y, Shen Y. 2011. Speckle reduction of ultrasound images based on Rayleigh-trimmed anisotropic diffusion filter. Pattern Recognition Letters 32(13):1516-1525.

Donoho D, Johnstone I. 1994. Ideal spatial adaptation via wavelet shrinkage. Biometrika 81, p. 425-455.

Donoho D, Johnstone I. 1995. Adapting to unknown smoothness via wavelet shrinkage. Journal of the American Statistical Association 90(432):1200-1224.

Finn S, Glavin M, Jones E. 2011. Echocardiographic speckle reduction comparison. IEEE Transactions on Ultrasonics, Ferroelectrics and Frequency Control 58(1):82-101. 

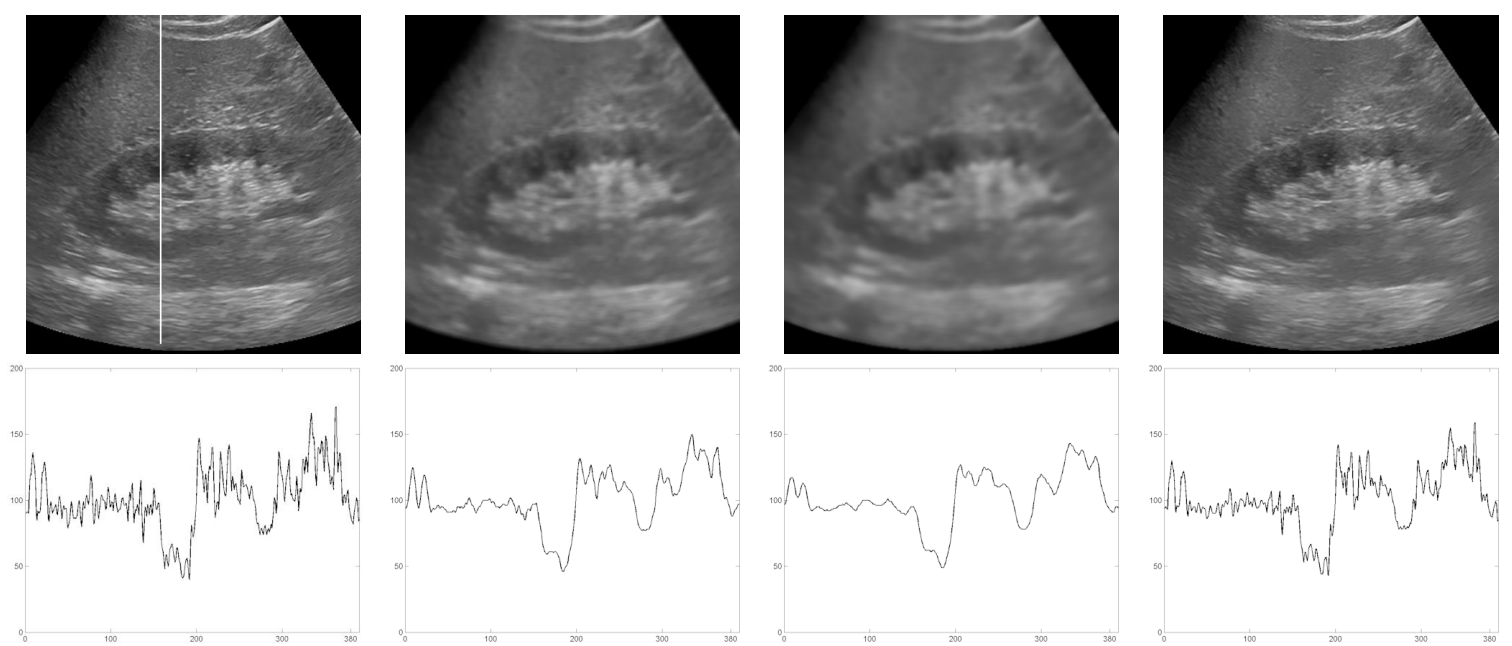

Original
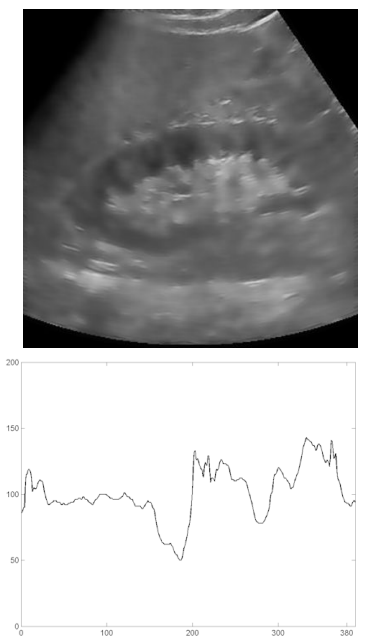

Wiener
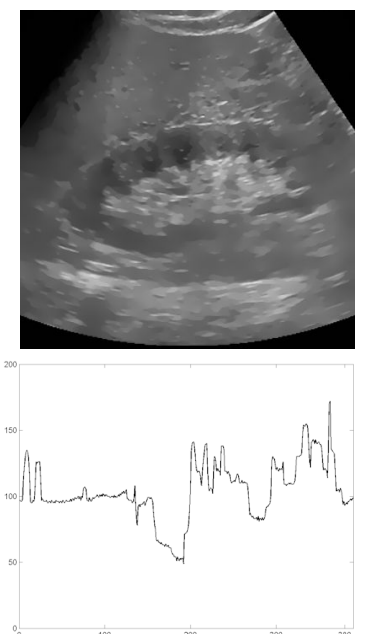

SRAD
Lee
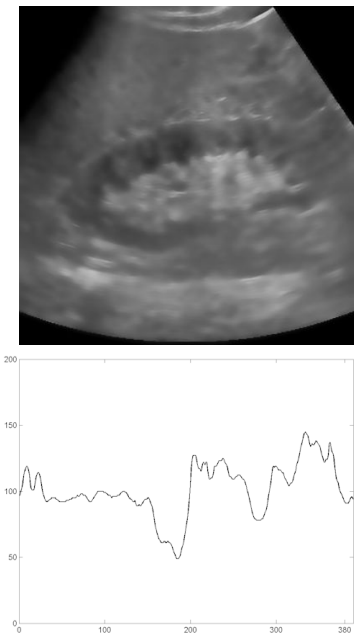

Bilateral
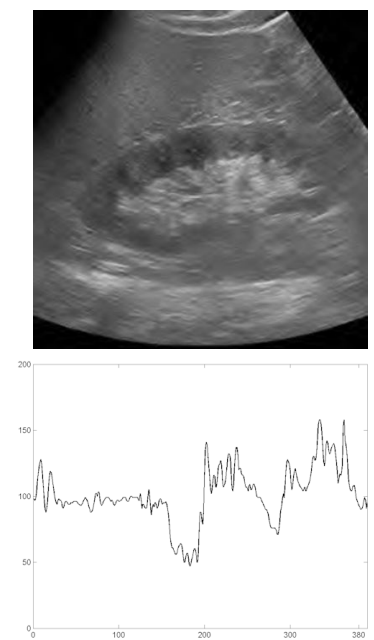

VisuShrink
Frost
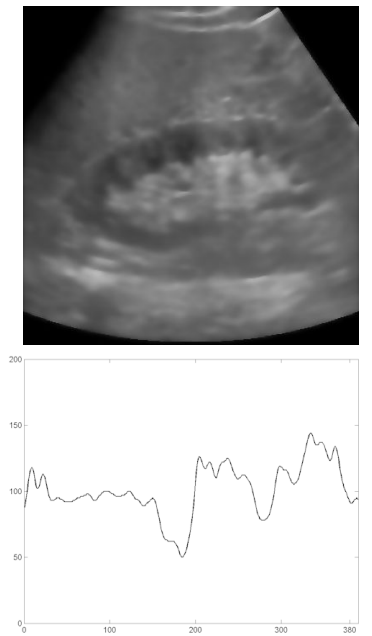

PMAD
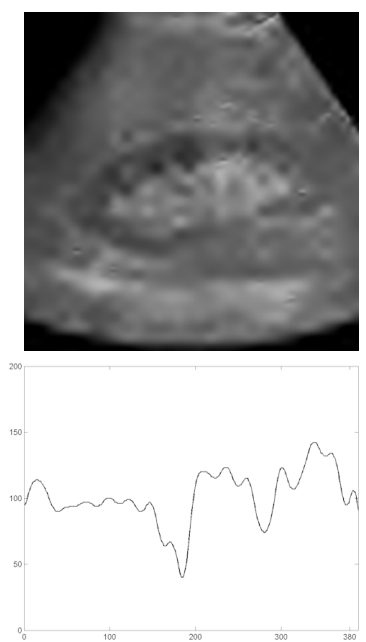

SureShrink
Kuan
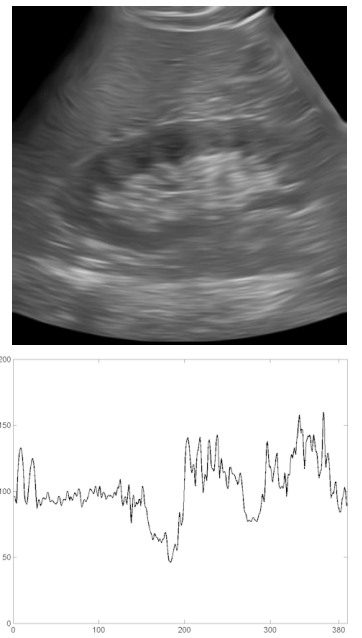

CED
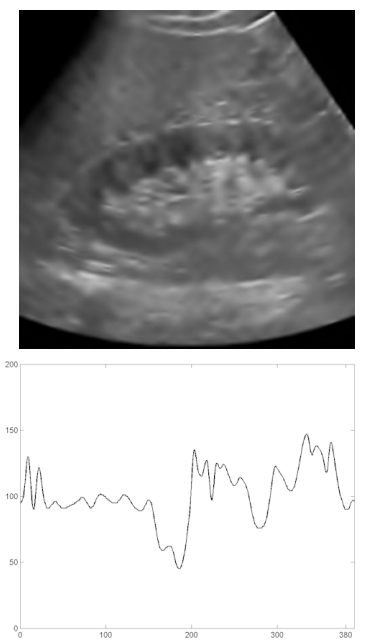

LMMSE

Figure 4. Original ultrasound image and the despecking filtered images with the corresponding profiles. 
Frost V, Stiles J, Shanmugan K, Holtzman J. 1982. A model for radar images and its application to adaptive digital filtering of multiplicative noise. IEEE Trans. Pat. Analysis and Machine Intelligence 4(2):157-166.

Fu S, Ruan Q, Fu S, Wang W, Li Y. 2005. A compound anisotropic diffusion for ultrasonic image denoising and edge enhancement. In IEEE International Symposium on Circuits and Systems, Vol. 3, p. $2779-2782$.

Huang J, Yang X. 2013. Fast reduction of speckle noise in real ultrasound images. Signal Processing 93(4):684-694.

Jensen J. 2004. Simulation of advanced ultrasound systems using Field II. In IEEE International Symposium on Biomedical Imaging, p. 636-639.

Jin F, Fieguth P, Winger L, Jernigan E. 2003. Adaptive Wiener filtering of noisy images and image sequences. In International Conference on Image Processing, Vol. 3, p. 349-352.

Khare A, Khare M, Jeong Y, Kim H, Jeon M. 2010. Despeckling of medical ultrasound images using Daubechies complex wavelet transform, Signal Processing 90(2):428439.

Krissian K, Westin C, Kikinis R, Vosburgh K. 2007. Oriented speckle reducing anisotropic diffusion. IEEE Transactions on Image Processing 16(5):1412-1424.

Kuan D, Sawchuk A, Strand T, Chavel P. 1985. Adaptive noise smoothing filter for images with signaldependent noise. IEEE Transactions on Pattern Analysis and Machine Intelligence 7(2):165-177.

Lee J. 1980. Digital image enhancement and noise filtering by use of local statistics. IEEE Transactions on Pattern Analysis and Machine Intelligence 2(2):165-168.

Loizou C, Pattichis C. 2008. Despeckle filtering algorithms and software for ultrasound imaging. Synthesis Lectures on Algorithms and Software for Engineering. Ed. Morgan \& Claypool Publishers.

Mateo JL, Fernandez-Caballero AF. 2009. Finding out general tendencies in speckle noise reduction in ultrasound images. Expert Systems with Applications 36(4):7786-7797.

Ortiz S, Chiu T, Fox M. 2012. Ultrasound image enhancement: A review. Biomedical Signal Processing and Control 7(5):419-428.

Perona P, Malik J. 1990. Scale-space and edge detection using anisotropic diffusion. IEEE Transactions on Pattern Analysis and Machine Intelligence, 12(7):629-639.

Pratt WK. 2007. Digital Image Processing - 4th Edition. John Wiley \& Sons.

Thakur A, Anand RS. 2005. Image quality based comparative evaluation of wavelet filters in ultrasound speckle reduction. Digital Signal Processing 15(5):455-465.

Tomasi C, Manduchi R. 1998. Bilateral filtering for gray and color images. In Sixth International Conference on Computer Vision, p. 839-846.

Wagner R, Smith S, Sandrik J, Lopez H. 1983. Statistics of speckle in ultrasound B-scans. IEEE Transactions on Sonics and Ultrasonics 30(3):156-163.

Wang Z, Bovik AC. 2002. A universal image quality index. IEEE Signal Processing Letters 9(3):81-84.

Wang Z, Bovik AC, Sheikh HR, Simoncelli EP. 2004. Image quality assessment: From error visibility to structural similarity. IEEE Transactions on Image Processing, 13(4):600-612.

Wang Z, Li Q. 2011. Information content weighting for perceptual image quality assessment. IEEE Transactions on Image Processing 20(5):1185-1198.

Weickert J. 1999. Coherence-enhancing diffusion filtering. Int. Journal on Computer Vision 31(2-3):111-127.

Yu J, Tan J, Wang Y. 2010. Ultrasound speckle reduction by a Susan-controlled anisotropic diffusion method. Pattern Recognition 43(9):3083-3092.

Yu Y, Acton ST. 2002. Speckle reducing anisotropic diffusion. IEEE Transactions on Image Processing 11(11):1260-1270.

Zhang D, Bao P, Wu X. 2005. Multiscale LMMSE-based image denoising with optimal wavelet selection. IEEE Transactions on Circuits and Systems for Video Technology, 15(4):469-481. 